\title{
Matching fingerface based on area under the highest peaks curve
}

\begin{abstract}
Passwords are no longer valid for accessing personal resources, because it is easy to forge them, in either small or big systems. Today $\hat{Q}$ challenge is to find other alternatives for passwords. Biometric is one of the ways, by mean of using human characteristics in accessing system resources. Fingerprint and face can be used to secure systems, because fingerprint is a unique, un-repeatable. Using it with face it can be widely acceptable. This paper present a model of fingerprint and face recognition to gain access to sensitive information for only authorized person. The method is based on recognition of the concatenated fingerprint and face images known as fingerface. A small size of fingerface images are used to extract information. Area under the curve resulted from connecting highest peaks are used in matching parameter. Other parameters are used to ensure a perfect matching. Our method takes into account a minimum image size and minimum number of searches. As a result we get fast in matching process.
\end{abstract}

Keyword: Fingerprint; Face; Hough transform; Area under curve; Peaks 